\title{
ANÁLISE DO GRAU DE SATISFAÇÃO NO TRABALHO EM EMPRESAS DA REGIÃO CENTRAL DO ESTADO DE RONDÔNIA
}

\section{ANALYSIS OF THE DEGREE OF SATISFACTION IN WORK IN COMPANIES IN THE CENTRAL REGION OF THE STATE OF RONDÔNIA}

\author{
Geva Franco Uchôa Nery1*, Ronaldo Helfenstein²
}

1 Acadêmica do 8ํ Período do Curso de Administração- Faculdade de Informática de Ouro Preto do Oeste- UNEURO.

2 Professor Orientador- Graduado em Administração pela Universidade Estadual do Oeste do ParanáUNIOESTE. Especialista em Marketing pela UNIOESTE e Mestre em Ciências Contábeis pela FUCAPE Business School. Professor dos cursos de Administração e Ciências Contábeis da Faculdade UNEOURO.

*Autor correspondente: gevafranco@hotmail.com

Recebido: 06/11/2017; Aceito: 09/12/2017

\section{RESUMO}

O objetivo deste artigo é analisar o grau de satisfação das pessoas no trabalho. A discussão teórica encontrou os modelos de Walton [1] e Siqueira [2] os quais foram adaptados e reunidos em um mesmo instrumento de levantamento de dados. Essa adaptação resultou em seis dimensões e 19 critérios de análise da qualidade de vida e satisfação no trabalho. Quarenta e sete pessoas de empresas privadas, instituições públicas e do terceiro setor participaram da pesquisa. Os resultados mostram que os fatores mais negativos são: oportunidades no trabalho e salário justo e adequado. Os fatores positivos são: trabalho e vida e integração social no trabalho.

Palavras-chave: Satisfação. Trabalho. Empregados.

\section{ABSTRACT}

The objective of this study is to aim the degree of satisfaction of people at work. The theoretical discussion found the models of Walton [1] and Siqueira [2] that were adapted and gathered in the same instrument of data collection. This adaptation resulted in six dimensions and 19 criteria for quality of life analysis and job satisfaction. Forty-seven people from private companies, public and third sector institutions participated in the survey. The results show that the most negative factors are opportunities at work and fair and adequate salary. The positive factors are work and life and social integration at work. Keywords: Satisfaction. Job. Employees

\section{INTRODUÇÃO}

$\mathrm{Na}$ atualidade, estudos sobre a satisfação no trabalho (ST) vêm se expandindo, pois as instituições têm encontrado nas pessoas uma importante fonte para seu crescimento. Dessa maneira, a importância da ST para a área de Recursos Humanos $(\mathrm{RH})$ tem sido expressiva.

Inicialmente este artigo aborda a ST, a classificação dos níveis de satisfação, suas causas fundamentais e suas consequências. Investiga as variáveis influentes na satisfação, como o salário, as condições de trabalho, o uso das capacidades dos indivíduos dentro da organização, as oportunidades que 
têm no local de trabalho, a integração social no trabalho e o espaço que o trabalho ocupa na vida do colaborador. Em seguida é apresentada a metodologia utilizada e a análise dos dados da pesquisa realizada de acordo com a adaptação feita sobre 0 método de Walton [1] que mensurava o grau de Qualidade de Vida no Trabalho, também foi utilizada para esta adaptação o modelo proposto por Siqueira [2]. Por fim, são apresentados os resultados.

A discussão teórica deste trabalho aborda sobre satisfação no trabalho. Tal assunto já foi estudado por diversos autores como Walton [1] e Siqueira [2] que desenvolveram seus modelos que foram adaptados para este trabalho. $O$ modelo proposto por Walton [1] aborda a Qualidade de Vida no Trabalho, e o modelo de Siqueira [2] estuda os fatores da Satisfação no Trabalho.

Esta discussão teórica traz aspectos como: níveis de ST, causas da ST e consequências da ST.

Diante disso, o presente artigo objetiva verificar o índice de satisfação no trabalho de trabalhadores da iniciativa privada, órgãos públicos e do terceiro setor. Fez-se oportuno entender, de forma teórica, os conceitos e variáveis que podem contribuir para o grau de satisfação.

\subsection{SATISFAÇÃO NO TRABALHO}

Segundo Siqueira [3], os primeiros estudos sobre a satisfação no trabalho eram considerados como um componente da motivação, que levava os $\begin{array}{llr}\text { trabalhadores a } & \text { apresentarem } \\ \text { indicadores de } & \text { comportamento }\end{array}$ importantes para o interesse empresarial, tais como o aumento do desempenho e da produtividade, permanência na empresa e redução de faltas ao trabalho. Nas décadas de 1970 e 1980, a satisfação no trabalho passou a ser uma característica da atitude, exigindo maior responsabilidade social por parte das empresas.

A satisfação no trabalho adentra 0 século XXI, como um dos múltiplos conceitos que abordam a afetividade no ambiente de trabalho ou mais especificamente, como um vínculo afetivo do indivíduo com seu emprego, sendo apontada como um dos três componentes psicossociais do conceito de bem-estar no trabalho, ao lado de envolvimento e comprometimento organizacional afetivo, (PADOVAM apud, SIQUEIRA [3]).

Satisfação no trabalho é algo de difícil definição, por se tratar de um estado subjetivo, podendo variar entre os indivíduos, de acordo com diferentes circunstâncias e ao longo do tempo.

Locke [4] definiu a satisfação no 
trabalho como um estado emocional, positivo ou de prazer, resultante de um trabalho ou de suas experiências definição que tem, ainda hoje, impacto relevante para o conceito.

Segundo Spector [5], a satisfação no trabalho é uma variável de atitude que mostra como as pessoas se sentem em relação ao seu trabalho, seja no todo, seja em relação a alguns de seus aspectos. "É O quanto as pessoas gostam dos seus trabalhos". Dessa forma, é necessário que a organização possa entender a satisfação do empregado como os valores, atitudes e objetivos pessoais que precisam ser identificados, compreendidos e estimulados para que estes consigam sua efetiva participação na empresa.

Torna-se fundamental que haja uma relação entre os objetivos organizacionais e os objetivos individuais dos colaboradores, para que os objetivos da organização sejam alcançados.

Para Spector [5], os empregados devem estar ligados ao desempenho individual, grupal e organizacional para que os objetivos da organização sejam alcançados. $O$ incentivo da empresa parece ser um caminho interessante para a satisfação dos funcionários [6]. Bergamini [6] também mostra que muitas vezes o fracasso das empresas, não está na falta de conhecimento técnico e sim na maneira como lidam com as pessoas.
A satisfação no trabalho traz reflexos e resultados em vários pontos da instituição. Spector [5] apresenta duas variáveis da correlação da satisfação no trabalho e com o desempenho. A primeira é que a satisfação ocasiona um bom desempenho, assim quando o trabalhador está satisfeito, ele pode desempenhar melhor e com mais prazer sua atividade. A segunda é oposta. $O$ desempenho ocasiona a satisfação. Porém Pereira [7] lembra que a produtividade e satisfação não se relacionam de uma forma linear.

\subsection{NÍVEIS DE SATISFAÇÃO NO TRABALHO}

De acordo com Spector [5], para o estudo da satisfação no trabalho existem duas abordagens: a abordagem global que, considera a satisfação no trabalho como um sentimento único, como um todo, apresentando um único índice geral da satisfação e a abordagem em facetas ou aspectos. Nesta abordagem, uma pessoa apresenta diferentes níveis de satisfação em relação às várias facetas. Dessa forma, a satisfação global pode ser interpretada como um índice geral da satisfação ou como um índice de diferentes facetas.

Zanelli et.al [8] apresentam a visão multidimensional, que é um conjunto de reações especificas a vários componentes do trabalho. Acrescentam 
que, em contrapartida, existem os estudiosos que defendem a visão unidimensional, que concebem a satisfação no trabalho como uma atitude geral ante o trabalho como um todo.

Zanelli et al. [8] mostram que as dimensões ou aspectos particulares no trabalho são diferentes, podendo variar de situação para situação. Por este motivo, a satisfação no trabalho é de difícil mensuração.

\subsection{CAUSAS DA SATISFAÇÃO NO TRABALHO}

Para Spector [5], a satisfação no trabalho é um produto de adequação entre o indivíduo e seu trabalho. $\mathrm{O}$ autor ainda propõe que o ambiente externo e a personalidade podem impactar na satisfação no trabalho, bem como sua influência conjunta.

Um fator que deve ser relevante e será demonstrado no presente artigo, é que os motivos que podem gerar satisfação em uma determinada cultura, não necessariamente são os mesmo fatores que geram satisfação em outras culturas. Spector [5] diz que pessoas apresentam sentimentos diferentes em relação ao trabalho, o isso envolve valores básicos que variam de local.

Inicialmente, faz-se a relação da satisfação no trabalho com o salário e seus benefícios, estes, considerados fatores que eliminam a insatisfação, mas não aumenta a capacidade de produção. Oliveira [9] diz que partindo do contrato de trabalho, o trabalhador despende energia para produzir em força de uma remuneração pelo trabalho, com a qual reproduz sua energia: o salário. Diante deste pensamento, entende-se que 0 salário e os benefícios são os métodos que o trabalhador possui para conseguir seus bens, suprindo assim as suas diversas necessidades.

Para o empregado estar satisfeito com o trabalho é necessário que ele esteja satisfeito também com seu chefe/superior.

Tanto para Bowditch e Buono [10] quanto para Robbins [11] o líder é quem tem a capacidade de influenciar pessoas para alcançarem determinada meta ou objetivo. O chefe deve saber conduzir, trazer motivação aos seus subordinados, pois isso pode refletir positiva ou negativamente nos índices da organização.

Segundo Robbins [11] trabalhos interessantes que fornecem treinamento, variedade, independência e controle, satisfaz a maioria dos funcionários. Desta forma, quanto mais a organização proporciona formas de crescimento e desenvolvimento, mais o empregado tende a ficar satisfeito com seu trabalho. Essas características estão ligadas ao fator que criam de fato satisfação e aumenta a produtividade. Encerra-se os 
fatores com a declaração de Bergamini [6] que afirma a substituição da remuneração pelo relacionamento interpessoal como fator de satisfação entre os funcionários, a ênfase no relacionamento interpessoal é, então, o mais direto substituto da remuneração por produção. Mostra assim, que um fator intrínseco pode proporcionar uma maior satisfação que fatores extrínsecos.

Um bom relacionamento interpessoal assegura um ambiente educado e afável [12]. Isso é benéfico, tanto para os colaboradores, quanto para à organização, proporcionando as pessoas, mais satisfação em trabalhar [12]. Segundo Moscovici [12] o relacionamento interpessoal e o clima influenciam-se recíproca e circularmente, caracterizando um ambiente agradável e estimulante ou desagradável, podendo causar a satisfação ou insatisfação, necessário para manter a relação entre os indivíduos na organização.

\subsection{CONSEQUÊNCIAS \\ SATISFAÇÃO NO TRABALHO}

DA

Segundo Henne e Locke [13] estar ou não satisfeito em relação ao trabalho, incorre em consequências diversas, sejam elas na área pessoal ou profissional, afetando diretamente 0 comportamento, a saúde e o bem-estar do trabalhador.

Rahman e Sem [14] afirmam que o absenteísmo é menor entre os que possuem uma maior satisfação no trabalho. Os autores também apontam que a satisfação no trabalho possui grande influência na determinação dos níveis de estresse e na qualidade de vida do trabalhador.

Alguns estudos relataram que a satisfação no trabalho apresenta consequências à saúde mental [14]. Rahman e Sem [14], realizaram uma pesquisa com 82 trabalhadores de uma fábrica, com idade média de 34 anos. Estes tinham uma média de 14 anos de experiência profissional, nível primário de educação e realizavam trabalho repetitivo. Esses autores encontraram associação significativa entre satisfação no trabalho e saúde mental [14]. Trabalhadores que tinham uma maior satisfação no trabalho estavam em um estado de saúde mental melhor, isso em comparação aos que tinham uma baixa satisfação no trabalho [14]. Além disso, quem estava com um melhor estado de saúde também tinha maior satisfação no trabalho [14].

Estudos de Caplan (et al.) e Roman \& Trice apud [14] também apontaram essa associação. Zalewska [15], estudou a importância dos relacionamentos sociais nos aspectos do trabalho e na satisfação no trabalho. Resultados mostram que a satisfação no trabalho conduz para uma melhor saúde 
física e mental [14] e relatos de menor incidência de doenças em trabalhadores satisfeitos $e$, consequentemente, uma melhor qualidade de vida dos mesmos [13]. O'Driscoll e Beehr [16] também encontraram associação estatisticamente significativa entre satisfação no trabalho e saúde mental, sendo que uma maior satisfação remete a um menor número de queixas de saúde. O quadro 1 representa um resumo dos modelos de Walton [1] e Siqueira [2].

Quadro 1: determinantes de motivação e satisfação no trabalho.

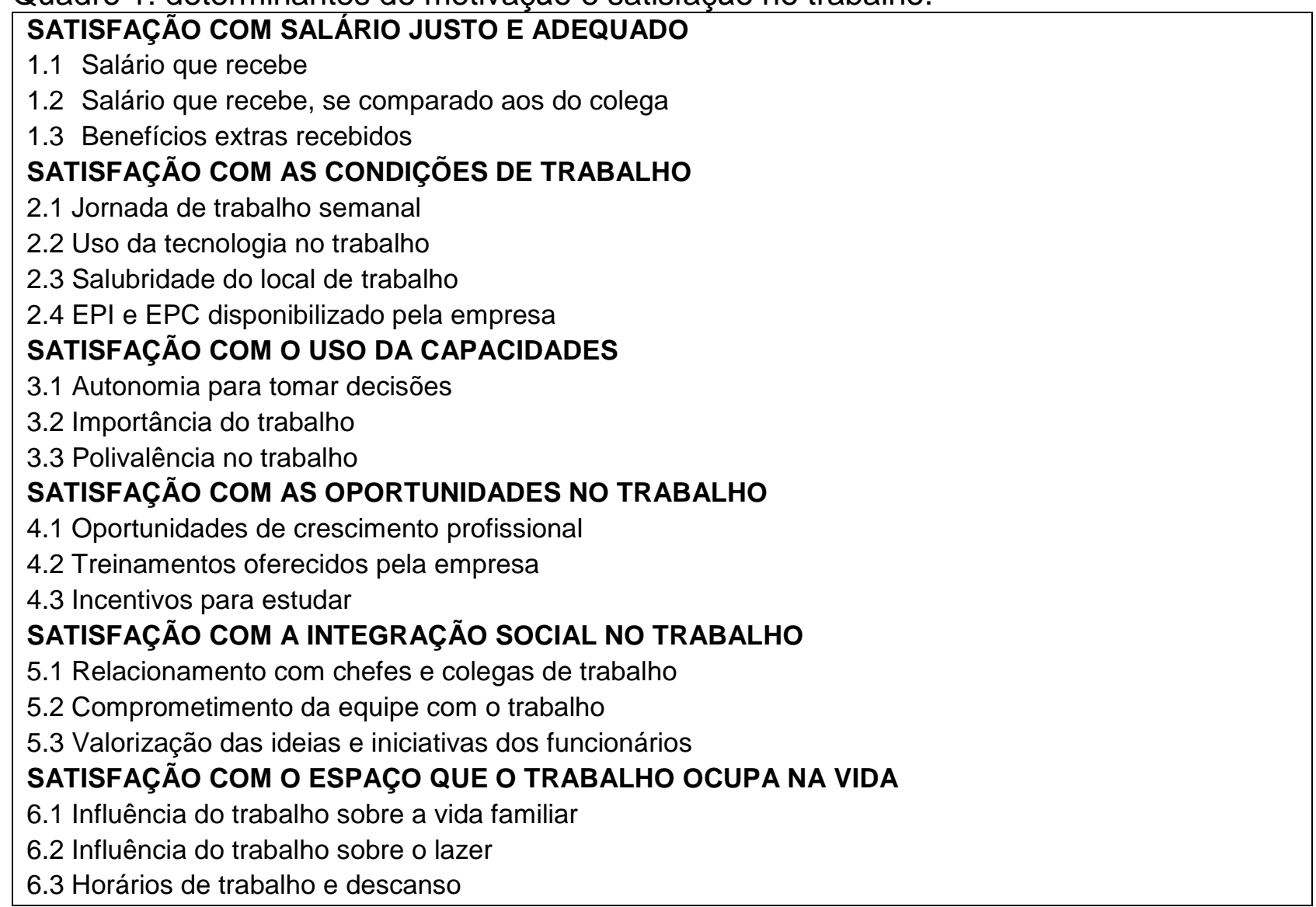

Fonte: adaptado de Walton [1] e Siqueira [2]

\section{METODOLOGIA}

Este artigo foi realizado por meio de uma pesquisa quantitativa e de caráter descritivo com corte transversal. Segundo Fonseca [17] a pesquisa quantitativa recorre a linguagem matemática para descrever as causas de um fenômeno, as relações entre variáveis. $\mathrm{O}$ caráter descritivo se reflete na descrição do fenômeno da satisfação no trabalho GIL [18]. O corte transversal é verificado porque a pesquisa apresenta dados de apenas um momento [18].

O questionário foi elaborado com base em Walton [1] e Siqueira [2]. Walton [1] definiu as variáveis da qualidade de vida no trabalho e Siqueira [2] elaborou as variáveis que foram aplicadas na medida da satisfação no trabalho. 0 
instrumento utilizado para entrevista foi aplicado pessoalmente.

Os itens apresentados no quadro 1, foram utilizados no questionário. Os modelos desses autores foram discutidos no capítulo que trata da fundamentação teórica e resultaram na construção do instrumento de levantamento de dados.

Esse instrumento foi submetido a um teste com quinze pessoas, resultando na exclusão de questões redundantes, tendenciosas, de difícil compreensão e/ou muito longas.

O questionário foi respondido através da escala Likert de cinco pontos (Muito insatisfeito, insatisfeito, nem insatisfeito/nem satisfeito, satisfeito e muito satisfeito), que foram respectivamente pontuados de 1 a 5 . A pontuação de cada dimensão equivale a soma das pontuações atribuídas de cada fator.

Quadro 2: escala de Likert:

\begin{tabular}{|c|c|c|c|c|}
\hline Muito Insatisfeito & Insatisfeito & $\begin{array}{c}\text { Nem Insatisfeito, } \\
\text { Nem Satisfeito }\end{array}$ & Satisfeito & Muito Satisfeito \\
\hline 1 & 2 & 3 & 4 & 5 \\
\hline
\end{tabular}

Fonte: Adaptado de Rueda [19]

Os resultados foram interpretados de acordo com os critérios do quadro 3 .

Quadro 3: interpretação dos resultados: Muito baixo: Até 1,5. Baixo: Maior que 1,5 até 2,5. Médio: Maior que 2,5 até 3,5. Alto: Maior que 3,5 até 4,5. Muito Alto: Maior que 4,5 até 5 . Fonte: elaborada pelos autores.

\section{RESULTADOS E DISCUSSÕES}

Participaram da pesquisa 47 pessoas. Os dados foram tratados de forma quantitativa, apresentados em forma de tabelas realizadas na planilha Excel, antecedendo de comentários.

\subsection{GRAU DE SATISFAÇÃO NO TRABALHO PARA AS SEIS DIMENSÕES}

A tabela 1 apresenta a média geral de cada dimensão. Observa-se, portanto, que a satisfação dos entrevistados é média para os fatores "salário justo e adequado"; "condições de trabalho" e "oportunidades no trabalho".

A satisfação é considerada alta para as dimensões "Uso das capacidades no trabalho"; "integração social no trabalho" e "trabalho e vida". 
Tabela 1: Análise dos resultados por dimensão

\begin{tabular}{|c|c|c|}
\hline DIMENSÃO & & MÉDIA \\
\hline 1- SALÁRIO JUSTO E ADEQUADC & & 3,035 \\
\hline 2- CONDIÇÕES DE TRABALHO & & 3,383 \\
\hline 3- USO DAS CAPACIDADES & NO & \\
\hline TRABALHO & & 3,532 \\
\hline OPORTUNIDADES & NO & \\
\hline TRABALHO & & 2,979 \\
\hline 5- INTEGRAÇÃO SOCIAL & NO & \\
\hline TRABALHO & & 3,606 \\
\hline 6- TRABALHO E VIDA & & 3,574 \\
\hline
\end{tabular}

Fonte: elaborada pela autora.

\subsection{NÍVEL DE SATISFAÇÃO NO} TRABALHO PARA DEZENOVE CRITÉRIOS AVALIADOS.

$\mathrm{Na}$ tabela 2, analisa-se a média por fator, proporcionando avaliar o grau de satisfação para cada item questionado. Esta tabela contribui para que o empregador tome conhecimento dos fatores que levam a insatisfação dos empregados, possibilitando a aplicação de medidas que deixem os colaboradores mais satisfeitos no ambiente laboral.

Observa-se que, o grau de satisfação baixo está ligado às oportunidades de crescimento profissional. Porém dentro da mesma dimensão "Oportunidades no trabalho" tem-se o grau de satisfação médio para incentivos aos estudos dos funcionários. Além disso, resultados mais específicos mostram que, as principais fontes de desapontamentos dos funcionários estão em benefícios extras recebidos e oportunidades de crescimento profissional.

Tabela 2: Análise dos resultados por fator

\begin{tabular}{lc}
\hline Fatores geradores de motivação e satisfação & Média \\
\hline SATISFAÇÃO COM SALÁRIO JUSTO E ADEQUADO & 3,08 \\
1.1 Salário que recebe & 3,23 \\
1.2 Salário que recebe, se comparado aos do colega & 2,78 \\
1.3 Benefícios extras recebidos & \\
SATISFAÇÃO COM AS CONDIÇÕES DE TRABALHO & 3,63 \\
2.1 Jornada de trabalho semanal & 3,34 \\
2.2 Uso da tecnologia no trabalho & 3,29 \\
2.3 Salubridade do local de trabalho & 3,25 \\
2.4 EPI e EPC disponibilizado pela empresa & 3,27 \\
SATISFAÇÃO COM O USO DA CAPACIDADES & 3,74 \\
3.1 Autonomia para tomar decisões & 3,57 \\
3.2 Importância do trabalho & 2,14 \\
3.3 Polivalência no trabalho & 2,93 \\
SATISFAÇÃO COM AS OPORTUNIDADES NO TRABALHO & 3,08 \\
4.1 Oportunidades de crescimento profissional & \\
4.2 Treinamentos oferecidos pela empresa & 3,70 \\
4.3 Incentivos para estudar & 3,36 \\
SATISFAÇÃO COM A INTEGRAÇÃO SOCIAL NO TRABALHO & 3,29 \\
5.1 Relacionamento com chefes e colegas de trabalho & \\
5.2 Comprometimento da equipe com o trabalho & 3,70 \\
5.3 Valorização das ideias e iniciativas dos funcionários & 3,42 \\
SATISFAÇÃO COM O ESPAÇO QUE O TRABALHO OCUPA NA VIDA & 3,59 \\
6.1 Influência do trabalho sobre a vida familiar &
\end{tabular}

Fonte: elaborada pelos autores. 
Os funcionários das empresas parecem gostar da jornada semanal de trabalho, consideram seus trabalhos importantes e, de modo geral, estão satisfeitos com os relacionamentos no trabalho. A respeito da vida pessoal dos funcionários, os resultados parecem mostrar que 0 trabalho afeta positivamente a vida familiar das pessoas e que estão satisfeitas com seus horários de trabalho e de descanso.

\section{CONSIDERAÇÕES FINAIS}

Este artigo procurou compreender - grau Satisfação no Trabalho. Desta maneira foram adaptados modelos de questionário para o levantamento de dados. Essa adaptação resultou em seis dimensões e 19 critérios de análise da qualidade de vida e satisfação no trabalho.

Quarenta e sete pessoas de empresas privadas, instituições públicas e do terceiro setor participaram da pesquisa. Os resultados foram organizados em duas tabelas. A primeira apresenta as estatísticas descritivas dos resultados para as seis dimensões, e a segunda, os resultados dos dezenove critérios. Os resultados foram interpretados de acordo com os seguintes critérios: até 1,5 satisfação muito baixa; maior que 1,5 até 2,5 satisfação baixa, maior que 3,5 até 4,5 , satisfação alta e satisfação maior que 4,5 foi considerada satisfação muito alta, desta forma, analisou-se os resultados que mostraram que os fatores mais negativos são: oportunidades no trabalho e salário justo e adequado. Os fatores positivos são: trabalho e vida e integração social no trabalho.

O presente artigo contribui para a área empresarial e de Recursos Humanos, pois demonstram resultados em relação aos indicadores em questão, é uma pesquisa com um número pequeno de participantes, todavia essas pessoas não são apenas de uma localidade do estado, apesar de ser um número reduzido, pode-se dizer que tornou-se abrangente pelo fato de se expandir por algumas cidades do estado. Porém, sugere-se que, novos estudos a respeito do tema discutido neste trabalho, sejam novamente realizados e que seja alterado a metodologia para corte horizontal e que a pesquisa se dê em uma única organização, desta forma acredita-se que 0 estudo realizado contribuirá ainda mais para a área empresarial, pois esta é uma área em movimento constante e precisa de mais de contribuições cientificas.

\section{REFERÊNCIAS}

[1] WALTON, R.E. Quality of working life is it? Slow Management Review, 1973. 
[2] SIQUEIRA, M.M.M. Antecedentes de comportamentos de cidadania organizacional: Análise de um modelo pós-cognitivo. Tese de Doutorado. Instituto de Psicologia Universidade de Brasília, 1995.

[3] SIQUEIRA, M.M.M. Satisfação no Trabalho. In: M.M.M. Siqueira (Org.). Medidas do comportamento organizacional. Porto Alegre: Artmed, 2008

[4] LOCKE, E.A. The Nature and Causes of Job Satisfaction. In: Dunnette, M.D., Ed., Handbook of Industrial and Organizational Psychology, 1976

[5] SPECTOR, Paul E. Psicologia nas organizações. São Paulo: Saraiva, 2003.

[6] BERGAMINI, Cecilia Whitaker. Motivação nas organizações. 5. ed. São Paulo: Atlas, 2008.

[7] PEREIRA, Orlindo Gouveia. Fundamentos do Comportamento Organizacional. Porto Alegre: Artmed, 2004.

[8] ZANELLI, José Carlos; BORGESANDRADE, Jairo E.; BASTOS, Antônio V. B. Psicologia, organizações e trabalho no Brasil. Porto Alegre: Artmed 2004.

[9] OLIVEIRA, L. T.; ZAMBERLAN, C. Satisfação no trabalho. Anais Jornada de Pesquisa Ulbra Santa Maria. Santa Maria, 2006.

[10] BOWDITCH, James L.; BUONO, Anthony $F$. Elementos do comportamento organizacional. São Paulo: Pioneira, 1992.

[11] ROBBINS, S. P.; JUDGE, T. A.; SOBRAL, F. Comportamento organizacional: teoria e pratica no contexto brasileiro. 14. ed. São Paulo: Pearson, 2010.
[12] MOSCOVICl, Fela. Desenvolvimento interpessoal: treinamento em grupo. 12. ed. Rio de Janeiro: José Olympio, 2002.

[13] HENNE, D.; \& LOCKE, E. Job dissatisfaction: what are the consequences? International Journal of Psychology, 1985

[14] RAHMAN, M.; \& SEM, A. K. Effect of job satisfaction on stress, performance and health in self-paced repetitive work. Int. Arch. Occup. Environ. Health, 1987.

[15] ZALEWSKA, A. M. Achievement and social relations values as conditions of the importance of work aspects and job satisfaction. Int. Occup. Saf. Ergon, 1999.

[16] O'DRISCOLL, M. P.; \& BEEHR, T. A. Moderating effects of perceived control and need for clarity on the relationship between role stressors and employee affective reactions. The Journal of Social Psychology., 2000.

[17] FONSECA, João José Saraiva da. Metodologia da pesquisa científica. Ceará: Universidade Estadual do Ceará, 2002.

[18] GIL, Antônio Carlos. Como elaborar projetos de pesquisa. 4. ed. São Paulo: Atlas, 2008.

[19] RUEDA, FABIAN XAVIER. Qualidade vida e satisfação no trabalho: Relação entre as escalas que avaliam os constructos. Universidade São Francisco. São Paulo. 2013. 